\title{
Perturbation Theory Approach to Rotational Tunneling Systems
}

\author{
Luciano T. Peixoto \\ Departamento de Fúsica \\ Universidade Federal do Espirito Santo \\ 29060-900 Vitoria, ES, Brazil
}

Received 5 October 1999

\begin{abstract}
Diagrammatic perturbation theory is used to consider the problem of the coupling of a molecular group libration to the lattice vibrations in solids exhibiting rotational tunneling detected by inelastic neutron scattering. The technique is applied to a molecular group of symmetry $C_{3}$ in presence of the hindering potential of the solid. The spectral density for transitions between rotational tunneling states in the ground librational level is obtained as a function of temperature. Low temperature results are presented for different phonon spectrum parameters. They are used to check the assumption that this coupling is the origin of the peculiar behaviour of the line shifting and broadening as a function of temperature in the INS spectra of such systems.
\end{abstract}

\section{Introduction}

The use of high-resolution neutron scattering apparatus has made possible to detect rotational tunneling states of molecular groups in solids. They are states arising from the quantum mechanical tunneling of the group between equivalent orientations in a hindering potential. The tunneling lifts the point group symmetry degeneracy of the librational levels and the neutron scattering process produces transitions among them (The term libration refers to the oscillation of an angular coordinate characterizing the orientation of the molecule.)

The explanation of the temperature dependence of the lines in the inelastic neutron scattering (INS) spectra from solids containing methyl and other similar groups was subject of controversy in the past $[1,2]$. From the so called thermometric point of view, the solid would work simply as a heat bath, and it would be the random jumps between equivalent orientations of the group that govern the peculiar temperature dependence of the lines[3, 4,5$]$. In contrast, a microscopic model has been proposed that accounts for the coupling of the molecular group to the lattice phonons. This spectrometric picture was applied to the problem of a molecular group of symmetry $C_{3}$ (like the methyl group) subjected to a deep potential barrier, first by considering the coupling to each potential well independently[6, 7$]$ and later by expressing the neutron scattering differential cross section in terms of time dependent correlation functions of standard basis operators for the rotational tunneling states[8, 9]. These correlation functions were calculated for a model with a linear coupling of the group to the lattice modes by using the Green function equation of motion technique. Low temperature expressions for the shifting and broadening of the lines corresponding to transitions between states in the ground librational level were obtained. The results reproduce the observed straight line for the broadening and an approximate straight line with smaller gradient for the shift when these quantities are plotted in a logarithmic scale against $1 / T$.

The low and high temperature regimes were studied[10] in the spectrometric picture by a path integral approach, and the results not only predict the same high temperature behavior as the classical hopping model but also confirm the low temperature calculations of the perturbation theory approach. More recently, a study using the generalized Langevin equation (the Nakajima-Zwanzing theory) for the spectral density projected in the sub-space of the tunneling states, succeeded to yield expressions for the line shift and line width from the Laplace transform of the frequency matrix and of the memory function, an approach which avoids the Random Phase Approximation implied in the former equation of motion treatment[11]. It is shown that these expressions reduce to those of reference [9] in the appropriate limits, and from them it is discussed how the phonon spectrum affects the INS spectra.

The criticism concerning the spectrometric model has been based on the fact that, compared with existing experimental data, their results reflect too much the details of the phonon spectrum. More recent experimental studies[2] of the methyl group in different environments but with nearly the same hindering potential barriers, show that instead of the coincident low 
temperature behavior of the linewidth expected from the thermometric picture, one has remarkable quantitative differences which do not happen at high temperatures. Although a qualitative agreement still persists, this shows clearly the limitations of the thermometric picture. Intriguing in this study, however, is the occurrence of an effective coincidence of the temperature dependence of the lineshift for some of the samples considered so that, although microscopically justified and in spite of its overall success, the spectrometric picture still has some points to clarify.

Our aim here is to improve the perturbation theory calculation for this problem, using a single molecular group approach to systems with molecules of symmetry $C_{3}$. We calculate the Green function by a diagrammatic perturbation expansion, including the contribution of the vertex part, instead of simply using the equation-of-motion formalism. This enables us to consider stronger coupling between rotational and vibrational states than in previous works, thus extending the range of parameter values for which the spectrometric theory can be tested. In particular we are interested to study the importance of the vertex part to explain experimental data. The main difficulty with the use of the diagramatic perturbation theory is with the lack of simple bosonic or fermionic commutation rules for the standard basis operators, implying that there is no such device as Wick's theorem to allow a direct evaluation of the diagrams. So we use the contour integral representation for thermal Green functions, adapting an existing diagrammatic technique[12]. In the spirit of the so called non-crossing approximation (NCA) we obtain a self-consistent set of coupled integral equations involving propagators, self-energies and vertex parts. From an iterative solution of these equations we construct the logarithmic plot of the linewidth and of the lineshift against $1 / T$ for different phonon spectrum parameters. The results confirm previous ones from the spectrometric picture and can be characterized, in view of the wider range of the parameters we were able to explore, as rather insensitive to the details of the phonon spectrum. At the same time we think that a valuable feature of this work is the development of the self-consistente calculation of the Green function with the vertex part contribution.

As we work here with a schematic form of the phonon spectrum, we think that any further advance on this problem will only happen on quantitative aspects by using phonon spectra corresponding to specific situations. On the other hand, the general problem of hindered rotation of the methyl group is under investigation in a variety of situations. A promising area has been initiated with the observation of rotational quantum tunneling of methyl in polymers[13]. The transition from quantum tunneling regime to classical random jump motion as the temperature is raised is a point not yet completely understood[14], and the effects of interaction between two or more mehtyl groups, as interpreted from observed level-crossing Nuclear Magnetic Resonance, is a subject under current investigation $[15,16,17]$.

\section{Description of the Calcula- tion}

The full Hamiltonian for the problem contains a term describing the interaction of the neutron beam with the solid which is responsible for transitions of the molecular groups between librational states split by quantum tunneling. Expressing this interaction as a superposition of standard basis operators, the differential neutron scattering cross section in the Born approximation can be obtained as a linear combination of time correlation functions of these operators. Using Fourier transform and integration in the complex plane, the line intensity corresponding to the transition $|m s\rangle \rightarrow\left|m^{\prime} s^{\prime}\right\rangle$ is given in proportion to the spectral density

$$
\rho_{m m^{\prime}}^{s s^{\prime}}(w)=\frac{1}{2 \pi i}\left[G_{m m^{\prime}}^{s s^{\prime}}(w-i \delta)-G_{m m^{\prime}}^{s s^{\prime}}(w+i \delta)\right],
$$

where $m$ is the librational quantum number and $s$ is the symmetry label indicating one of the irreducible representations $A, E^{a}$ and $E^{b}$ of the symmetry group $C_{3}$. In the contour integral representation, the Green's functions are the analytical continuation of[12]

$$
G_{m m^{\prime}}^{s s^{\prime}}\left(i w_{n}\right)=\frac{1}{2 \pi i Z} \oint d z \exp (-\beta z) \operatorname{Tr}\left[R(z) X_{m m^{\prime}}^{s s^{\prime}} R\left(z+i w_{n}\right) X_{m^{\prime} m}^{s^{\prime} s}\right],
$$

where $Z=\operatorname{Tr} \exp (-\beta H)$ is the partition function, $w_{n}$ is the Matsubara frequency, and $R(z)=(z-H)^{-1}$ is the resolvent. The $X_{m m^{\prime}}^{s s^{\prime}}=\left|m s><m^{\prime} s^{\prime}\right|$ are standard basis operator and the contour is chosen to encircles all the singularities of the integrand. The solid is described in terms of the set of rotational tunneling states of the moleculatr groups indicated in Fig. 1 coupled to the phonon states, with the Hamiltonian

$$
H=\sum_{m s} E_{s}^{m} X_{m m}^{s s}+\sum_{q} \hbar w_{q} b_{q}^{+} b_{q}+\sum_{q s} \sum_{m m^{\prime}} g_{m m^{\prime}}(q)\left(b_{q}^{+}+b_{q}\right) X_{m m^{\prime}}^{s s}
$$




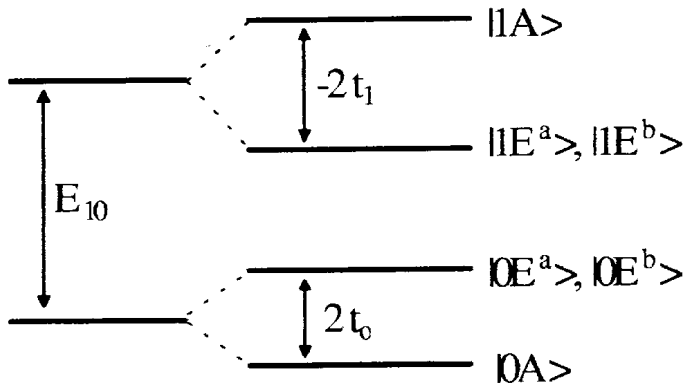

Figure 1. The energy level scheme corresponding to the two lowest rotational levels of a molecular group of symmetry $C_{3}$.

Here $E_{s}^{m}$ are the librational energies and $b_{q}^{+}$and $b_{q}$ are phonon creation and annihilation operators. The first two terms constitute the non interacting Hamiltonian with eigenstates given by the products $|m, s\rangle \mid$ $\left.\left\{n_{q}\right\}\right\rangle$. The third term represents the second quantized form of the coupling $\hat{V}$ between librational and vibrational levels which can be shown by symmetry arguments to occur only between librational levels of the same symmetry. For large enough potential barriers the coupling is also independent of the symmetry type[8], as already implicit in expression (03). Then $g_{m m^{\prime}}(q)$ is the matrix element $g_{m m^{\prime}}$ of $\hat{V}$ between the states $\mid m s>$ and $\mid m^{\prime} s>$ divided by $\sqrt{2 w_{q}}$. The assumption of large potential barriers implies large separation between rotational states so that for low enough temperatures we consider the transitions induced by the phonons only from the ground to the first rotational level. We are thus left with a double two level system, one composed by the states $|0 A\rangle$ and $|1 A\rangle$, with energies $E_{A}^{0}$ and $E_{A}^{1}$ respectively, and the other by the degenerate pairs $\left|0 E^{a}>,\right| 0 E^{b}>$ and $\left.\left|1 E^{a}>,\right| 1 E^{b}\right\rangle$, with energies $E_{E}^{0}$ and $E_{E}^{1}$ respectively (see Fig. 1 ).

We consider here only the absorption line, corresponding to the transitions $|0 A>\rightarrow| 0 E>$ induced by the neutron scattering process.With appropriate changes it is easy to calculate the emission line $\mid 0 E>\rightarrow$ $\mid 0 A>$ and the elastic line $\left|0 E^{a}>\rightarrow\right| 0 E^{b}>$. Evaluating the trace in the Green function,

$$
G_{00}^{A E}\left(i w_{n}\right) \equiv G\left(i w_{n}\right)=\frac{Z_{v}}{2 \pi i Z} \oint d z \exp (-\beta z) I\left(z, z^{\prime}\right)
$$

with $z^{\prime}=z+i w_{n}$ and

$$
\begin{aligned}
I\left(z, z^{\prime}\right)= & \frac{1}{Z_{v}} \sum_{k} e^{-\beta E_{k}}\left[\Re_{k k}^{0 A}\left(z+E_{k}\right) \Re_{k k}^{0 E}\left(z^{\prime}+E_{k}\right)\right. \\
& \left.+\sum_{k^{\prime} \neq k} \Re_{k k^{\prime}}^{0 A}\left(z+E_{k}\right) \Re_{k^{\prime} k}^{0 E}\left(z^{\prime}+E_{k}\right)\right]
\end{aligned}
$$

where $Z_{v}$ is the partition function for the vibrational system. The sum is over vibrational states with energies $E_{k}$ and in the matrix elements $\Re_{k k^{\prime}}^{m s}\left(z+E_{k}\right) \equiv<$ $m s k\left|\left(z+E_{k}-H\right)^{-1}\right| m s k^{\prime}>$ we have made the energy shift $z \rightarrow z+E_{k}$. Now using the identity

$$
\frac{1}{z+E_{k}-H}=\frac{1}{z+E_{k}-H_{0}}+\frac{1}{z+E_{k}-H_{0}} V \frac{1}{z+E_{k}-H}
$$

we obtain

$$
\Re_{k k^{\prime}}^{0 s}\left(z+E_{k}\right)=\frac{1}{z+E_{s}^{0}-\Im_{k k}^{0 s}\left(z+E_{k}\right)}\left[\delta_{k k^{\prime}}+\sum_{k^{\prime \prime} \neq k} \Im_{k k^{\prime}}^{0 s}\left(z+E_{k}\right) \Re_{k^{\prime \prime} k^{\prime}}^{0 s}\left(z+E_{k}\right)\right],
$$

where we will use the notation $R_{k k}^{0 s}\left(z+E_{k}\right)$ for the expression before the bracket and

$$
\Im_{k k^{\prime}}^{0 s}\left(z+E_{k}\right)=\sum_{k^{\prime \prime}} \frac{<s 0 k|V| k^{\prime \prime} 1 s><s 1 k^{\prime \prime}|V| k^{\prime} 0 s>}{z+E_{k}-E_{k^{\prime \prime}}-E_{s}^{1}}
$$

is the second order approximation for the self-energy. In the substitution of (06) in (05) we use the truncated form

$$
\begin{gathered}
\Re_{k k}^{0 s}\left(z+E_{k}\right)=R_{k k}^{0 s}\left(z+E_{k}\right) \\
\Re_{k k^{\prime}}^{0 s}\left(z+E_{k}\right)=R_{k k}^{0 s}\left(z+E_{k}\right) \Im_{k k^{\prime}}^{0 s}\left(z+E_{k}\right) R_{k^{\prime} k^{\prime}}^{0 s}\left(z+E_{k}\right)
\end{gathered}
$$

from which

$$
\begin{gathered}
I\left(z, z^{\prime}\right)=\frac{1}{Z_{v}} \sum_{k} \exp \left(-\beta E_{k}\right) R_{k k}^{0 A}\left(z+E_{k}\right) \Gamma_{k k}^{0}\left(z+E_{k}, z^{\prime}+E_{k}\right) R_{k k}^{0 E}\left(z^{\prime}+E_{k}\right) \\
\Gamma_{k k}^{0}\left(z+E_{k}, z^{\prime}+E_{k}\right)=1+\sum_{k^{\prime} \neq k} \Im_{k k^{\prime}}^{0 A}\left(z+E_{k}\right) R_{k^{\prime} k^{\prime}}^{0 A}\left(z+E_{k}\right) R_{k^{\prime} k^{\prime}}^{0 E}\left(z^{\prime}+E_{k}\right) \Im_{k^{\prime} k}^{0 E}\left(z^{\prime}+E_{k}\right) .
\end{gathered}
$$


The second term in (08-b) represents the vertex correction. The expression (08-a) is an average over vibrational states of a product of dressed propagators and self-energies. Considering the expansion

$$
R\left(z+E_{k}\right)=\frac{1}{z+E_{k}-H_{0}} \sum_{n=0}^{\infty}\left[\frac{\Im\left(z+E_{k}\right)}{z+E_{k}-H_{0}}\right]^{n}
$$

in all matrix elements of $R\left(z+E_{k}\right)$, the calculation reduces, term by term in the product of expansions, to the average of products of free propagators and self-energies. Introducing the occupation number representation, this transforms into a product of averages over phonon modes, and after some calculation we find the set of expressions

$$
\begin{gathered}
I\left(z, z^{\prime}\right)=R_{A}^{0}(z) \Gamma\left(z, z^{\prime}\right) R_{E}^{0}\left(z^{\prime}\right) \\
R_{s}^{m}(z)=\frac{1}{z-E_{s}^{m}-\Im_{s}^{m}(z)} \\
\Gamma\left(z, z^{\prime}\right)=1+\sum_{p n_{p} q n_{q}}\left|g_{p}\right|^{2} \frac{e^{-\beta n_{p} w_{p}}}{Z_{p}} \frac{\left.<n_{p}^{\prime} \mid b_{p}^{+}+b_{p}\right) \mid n_{p}>}{z-E_{A}^{1}+\left(n_{p}-n_{p}^{\prime}\right) w_{p}} \times \Gamma_{p q} \times \\
\mathrm{x}\left[\frac{1}{z^{\prime}-E_{E}^{1}+\left(n_{p}-n_{p}^{\prime}\right) w_{p}}+\frac{1}{z^{\prime}-E_{E}^{1}+\left(n_{q}-n_{q}^{\prime}\right) w_{q}}\right]
\end{gathered}
$$

with

$$
\begin{gathered}
\Gamma_{p q}=\left|g_{q}\right|^{2} \frac{e^{-\beta n_{q} w_{q}}}{Z_{q}} \frac{\left.\left.<n_{p} \mid b_{p}^{+}+b_{p}\right)\left|n_{p}^{\prime}><n_{q}\right| b_{q}^{+}+b_{q}\right) \mid n_{q}^{\prime}>}{z-E_{A}^{0}+\left(n_{p}-n_{p}^{\prime}\right) w_{p}+\left(n_{q}-n_{q}^{\prime}\right) w_{q}} \\
x \frac{\left.\left.<n_{q}^{\prime} \mid b_{q}^{+}+b_{q}\right)\left|n_{q}><n_{p}^{\prime}\right| b_{p}^{+}+b_{p}\right) \mid n_{p}>}{z^{\prime}-E_{E}^{0}+\left(n_{p}-n_{p}^{\prime}\right) w_{p}+\left(n_{q}-n_{q}^{\prime}\right) w_{q}} \\
\Im_{s}^{0}(z)=\sum_{q} \frac{\left|g_{q}\right|^{2}}{Z_{q}} \sum_{n_{q} n_{q}^{\prime}} e^{-\beta n_{q} w_{q}} \frac{\left.\left.<n_{q} \mid b_{q}^{+}+b_{q}\right)\left|n_{q}^{\prime}><n_{q}^{\prime}\right| b_{q}^{+}+b_{q}\right) \mid n_{q}>}{z-E_{s}^{1}+\left(n_{q}-n_{q}^{\prime}\right) w_{q}} \\
Z_{q}=\sum \exp \left(-\beta n_{q} w_{q}\right) .
\end{gathered}
$$

We are using $g_{q}$ as a shorthand notation for $g_{01}\left(w_{q}\right)$. The expression (11) can be viewed as the lowest order term in a product of dressed resolvents. With evaluation of the matrix elements it has the diagrammatic representation showed in Fig. 2, where the dashed and the full straight lines represent free propagators for the torsional levels $|0 A\rangle$ and $|0 E\rangle$ respectively. The curved lines represent the phonon propagators with diagrams for opposite directions of the phonon lines combined into one. In the NCA approximation we neglect the second type of diagrams and the remaining one can be viewed as the second term of a ladder expansion consistent with the NCA. The renormalization procedure within the NCA corresponds to a partial sum leading to the diagrams sequence of Fig 3 .

Note that the propagators for the librational states appear renormalized as well. With replacement of the sum over the phonon modes by an integral over phonon frequencies, introducing the density of phonon modes $\rho\left(w_{q}\right)$, the renormalization process corresponds to solve iteratively the pair of coupled integral equations for the vertex correction

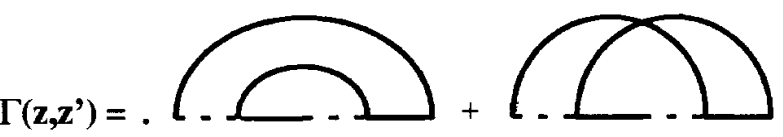

Figure 2. Diagramatic expansion corresponding to the expression (11)

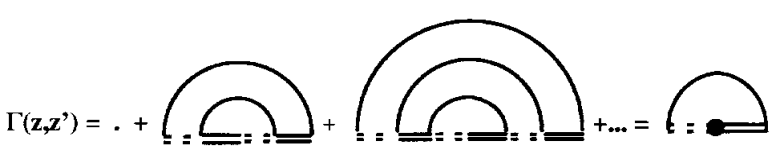

Figure 3. Diagramatic renormalization of expression (11) in the NCA approximation. 


$$
\begin{gathered}
\Gamma^{0}\left(z, z^{\prime}\right)=1+\int_{-\infty}^{\infty} R_{A}^{1}\left(z+w_{q}\right) \Gamma^{1}\left(z+w_{q}, z^{\prime}+w_{q}\right) R_{E}^{1}\left(z^{\prime}+w_{q}\right) g\left(w_{q}\right) d w_{q} \\
\Gamma^{1}\left(z, z^{\prime}\right)=\int_{-\infty}^{\infty} R_{A}^{0}\left(z+w_{q}\right) \Gamma^{0}\left(z+w_{q}, z^{\prime}+w_{q}\right) R_{E}^{0}\left(z^{\prime}+w_{q}\right) g\left(w_{q}\right) d w_{q}
\end{gathered}
$$

where $g\left(w_{q}\right)=g_{q}^{2} f\left(w_{q}\right) \rho\left(w_{q}\right)$, with $f\left(w_{q}\right)=\left\langle b_{q}^{+} b_{q}\right\rangle$ being the bose function. To reduce the length of the expressions we have imposed the condition $\rho\left(-w_{q}\right)=\rho\left(w_{q}\right)$ and extended the range of integration to include negative frequencies. The same reasoning applied to the self energies yields

$$
\Im_{s}^{m}(z)=\int_{-\infty}^{\infty} R_{s}^{m}\left(z+w_{q}\right) g\left(w_{q}\right) d w_{q}
$$

and with (10) we have the complete set of equations for the problem.

From the same equation (10) we see that the poles of $G\left(i w_{n}\right)$ are $\varepsilon_{A}^{0}$ and $\varepsilon_{E}^{0}-i w_{n}$ satisfying the equation

$$
\varepsilon_{s}^{m}=E_{s}^{m}+\operatorname{Re} \Im_{s}^{m}\left(\varepsilon_{s}^{m}\right)
$$

From the residue theorem and using $[12] \exp \left(i w_{n}\right)=-1$, we find from equation $(04)$

$$
\begin{aligned}
G\left(i w_{n}\right)= & \frac{Z_{v}}{Z}\left[e^{-\beta \varepsilon_{A}^{0}} \Gamma^{0}\left(\varepsilon_{A}^{0}, \varepsilon_{A}^{0}+i w_{n}\right) R_{E}^{0}\left(\varepsilon_{A}^{0}+i w_{n}\right) S_{A}^{0}-\right. \\
& \left.e^{-\beta \varepsilon_{E}^{0}} R_{A}^{0}\left(\varepsilon_{E}^{0}-i w_{n}\right) \Gamma^{0}\left(\varepsilon_{E}^{0}-i w_{n}, \varepsilon_{E}^{0}\right) S_{E}^{0}\right]
\end{aligned}
$$

with

$$
S_{s}^{0}=\left[1-\left|\frac{d}{d \varepsilon} \operatorname{Re} \Im_{s}^{0}(\varepsilon)\right|_{\varepsilon_{s}^{0}}\right]^{-1}
$$

Inserting the analytical continuation $i w_{n} \rightarrow w+i \delta$ we have from (04) the result

$$
\begin{aligned}
\rho(w)= & \frac{Z_{v}}{\pi Z}\left\{e^{-\beta \varepsilon_{A}^{0}}\left[X_{E}^{0}\left(\varepsilon_{A}^{0}+w\right) V_{E}^{0}\left(\varepsilon_{A}^{0}+w\right)+Y_{E}^{0}\left(\varepsilon_{A}^{0}+w\right) U_{E}^{0}\left(\varepsilon_{A}^{0}+w\right)\right] S_{A}^{0}+\right. \\
& \left.e^{-\beta \varepsilon_{E}^{0}}\left[X_{A}^{0}\left(\varepsilon_{E}^{0}-w\right) V_{A}^{0}\left(\varepsilon_{E}^{0}-w\right)+Y_{A}^{0}\left(\varepsilon_{E}^{0}-w\right) U_{A}^{0}\left(\varepsilon_{E}^{0}-w\right)\right] S_{E}^{0}\right\}
\end{aligned}
$$

where

$$
\begin{gathered}
U_{s}^{m}(x)=\delta_{0, m}+\int_{-\infty}^{\infty}\left[X_{s}^{n}\left(x+w_{q}\right) U_{s}^{n}\left(x+w_{q}\right)\right. \\
\left.-Y_{s}^{n}\left(x+w_{q}\right) V_{s}^{n}\left(x+w_{q}\right)\right] R_{s^{\prime}}^{n}\left(\varepsilon_{s^{\prime}}^{0}+w_{q}\right) g\left(w_{q}\right) d w_{q} \\
V_{s}^{m}(x)=\int_{-\infty}^{\infty}\left[X_{s}^{n}\left(x+w_{q}\right) V_{s}^{n}\left(x+w_{q}\right)\right. \\
\left.\quad+Y_{s}^{n}\left(x+w_{q}\right) U_{s}^{n}\left(x+w_{q}\right)\right] R_{s^{\prime}}^{n}\left(\varepsilon_{s^{\prime}}^{0}+w_{q}\right) g\left(w_{q}\right) d w_{q} \\
X_{s}^{m}(x) \pm i Y_{s}^{m}(x)=\frac{\left[x-E_{s}^{m}-A_{s}^{m}(x)\right] \pm i B_{s}^{m}(x)}{\left[x-E_{s}^{m}-A_{s}^{m}(x)\right]^{2}+\left[B_{s}^{m}(x)\right]^{2}} \\
A_{s}^{m}(x) \pm i B_{s}^{m}(x)=\int_{-\infty}^{\infty}\left[X_{s}^{n}\left(x+w_{q}\right) \pm i Y_{s}^{n}\left(x+w_{q}\right)\right] g\left(w_{q}\right) d w_{q} .
\end{gathered}
$$


In these expressions, $m=1$ when $n=0$ and viceversa, and also $U=\operatorname{Re}(\Gamma), V=\operatorname{Im}(\Gamma), X=\operatorname{Re}(R)$, $Y=\operatorname{Im}(R), A=\operatorname{Re}(\Im)$ and $B=\operatorname{Im}(\Im)$. Note that the input value of $x$ in these expressions when $m=0$ is the output value $x=x+w_{q}$ when $m=1$, and viceversa. In this way, when solving this system by iteration, we are in each step adding a new variable $w_{q}$ to the argument. According to (16), there are two starting expressions for $x: x=\varepsilon_{A}^{0}+w\left(s \equiv E, s^{\prime} \equiv A\right)$ and $x=\varepsilon_{E}^{0}-w\left(s \equiv A, s^{\prime} \equiv E\right)$.

\section{Solving the Equations: the Phonon Spectrum}

To solve this set of equations we rename the integrands of (17-a,b) and introduce variables $u_{0}$ and $u_{1}$ and functions $C$ and $D$, doubling the number of equations, and working with the set

$$
\begin{gathered}
U_{s}^{m}\left(u_{m}\right)=\delta_{0, m}+\int_{-\infty}^{\infty} C_{s}^{n}\left(u_{n}\right) f\left(u_{n}-u_{m}\right) d u_{n} \\
V_{s}^{m}\left(u_{m}\right)=\int_{-\infty}^{\infty} D_{s}^{n}\left(u_{n}\right) f\left(u_{n}-u_{m}\right) d u_{n} \\
C_{s}^{n}\left(u_{m}\right)=X_{s}^{n}\left(u_{m}\right) U_{s}^{n}\left(u_{n}\right)-Y_{s}^{n}\left(u_{m}\right) V_{s}^{n}\left(u_{n}\right) \\
D_{s}^{n}\left(u_{n}\right)=X_{s}^{n}\left(u_{n}\right) V_{s}^{n}\left(u_{n}\right)+Y_{s}^{n}\left(u_{n}\right) U_{s}^{n}\left(u_{n}\right)
\end{gathered}
$$

where $n=1$ when $m=0$ and vice-versa. From the Lorentzian shape (and of its derivative) of the function $Y_{s}^{m}$ (and $X_{s}^{m}$ ) we see that each of the functions $C_{s}^{n}$ and $D_{s}^{n}$ is different from zero only in a limited interval of its argument, centered at $\varepsilon_{s}^{0}$ or $\varepsilon_{s}^{1}$, each with a characteristic energy scale. We exploit this fact and also that the phonon density of states, through the function $f(w)$, introduces a cutoff at $w=w_{c}$. So, when choosing the grid of values for the variables to perform the numerical integrations, we are selecting a discrete and finite set of values of the functions $U, V, C$ and $D$, defined at these grid points and corresponding to characteristic energy increments $\Delta u_{n}$. Then the iteration process begins with a initial set of values for $U^{0}$ and $V^{0}$ (the natural choice is 1 for all $U^{0}$ and 0 for all $V^{0}$ ), using them in $(19-\mathrm{c}, \mathrm{d})$ to find the set of $C^{0}$ and $D^{0}$, which are substituted in $(19-\mathrm{a}, \mathrm{b})$ to find now the set of $U^{1}$ and $V^{1}$, then in (19-c,d) to find the set of $C^{1}$ and $D^{1}$ and then back in $(19-\mathrm{a}, \mathrm{b})$ to find new set of values of $U^{0}$ and $V^{0}$. The process continues to any desired order of approximation and it is its convergence that will validate the assumptions on the behavior of the functions and the choice of the grid increment of the variables. At the end, the spectral density is obtained from

$$
\rho(w)=\frac{Z_{v}}{\pi Z}\left[e^{-\beta \varepsilon_{A}^{0}} C_{E}^{0}\left(\varepsilon_{A}^{0}+w\right)+e^{-\beta \varepsilon_{E}^{0}} C_{A}^{0}\left(\varepsilon_{E}^{0}-w\right)\right] .
$$

The discrete set of values of the functions $X$ and $Y$, defined for the same grid points of the variables and used in the above equations, has to be found previously, from the corresponding system

$$
\begin{gathered}
A_{s}^{m}\left(u_{m}\right)=\int_{-\infty}^{\infty} X_{s}^{n}\left(u_{n}\right) g\left(u_{n}-u_{m}\right) d u_{n} \\
B_{s}^{m}\left(u_{m}\right)=\int_{-\infty}^{\infty} Y_{s}^{n}\left(u_{n}\right) g\left(u_{n}-u_{m}\right) d u_{n} \\
X_{s}^{m}\left(u_{m}\right)=\frac{u_{m}-E_{s}^{m}-A_{s}^{m}\left(u_{m}\right)}{\left[u_{m}-E_{s}^{m}-A_{s}^{m}\left(u_{m}\right)\right]^{2}+\left[B_{s}^{m}\left(u_{m}\right)\right]^{2}} \\
Y_{s}^{m}\left(u_{m}\right)=\frac{B_{s}^{m}\left(u_{m}\right)}{\left[u_{m}-E_{s}^{m}-A_{s}^{m}\left(u_{m}\right)\right]^{2}+\left[B_{s}^{m}\left(u_{m}\right)\right]^{2}}
\end{gathered}
$$

As above, the index $s$ distinguishes two set of equations, one for the states $A$ and the other for the states $E$, and again we have $n=1$ when $m=0$ and vice-versa. Note that the energies $\varepsilon_{A}^{m}$ and $\varepsilon_{E}^{m}$ are obtained for each temperature from the maximum of the expression (21-d) for $s \equiv A$ and $s \equiv E$, respectively. In this way, contrary to what is implicit in previous treatments, we do not work here with approximate expressions for the line width and the line shift of the tunneling states, but rather determine them self consistently, using the results to find the INS line behavior from the half-width and the point of maximum of the function $\rho(w)$.

The function $C$ or $D$ appearing in (19) is simply the term in brackets in the corresponding expressions (17), so that the function $f(x)$ in (19) is the product of $g(x)$ by the corresponding propagator $R_{s^{\prime}}^{n}\left(\varepsilon_{s^{\prime}}^{0}+w_{q}\right)$, which is real. We use here the approximation $R_{s^{\prime}}^{n}\left(\varepsilon_{s^{\prime}}^{0}+w_{q}\right) \approx$ $\left[w_{q}-\left(\varepsilon_{s^{\prime}}^{n}-\varepsilon_{s^{\prime}}^{0}\right)\right]^{-1}$ which implies that we can also take $S_{s}^{0}=1$ in (16). In other words, we have

$$
f\left(u_{n}-u_{m}\right)=\frac{g\left(u_{n}-u_{m}\right)}{u_{n}-u_{m}-\left(\varepsilon_{s}^{1}-\varepsilon_{s}^{0}\right)} .
$$

The problem with the singularity in $f(x)$ is dealt with 


$$
\begin{aligned}
A_{s}^{m}\left(u_{m}\right)= & \int_{u_{m}-w}^{u_{m}+w} F\left(u_{n}\right) \frac{g\left(u_{n}-u_{m}\right) d u_{n}}{u_{n}-u_{m}-\delta \varepsilon}=\int_{u_{m}-w}^{u_{m}+w} \frac{F\left(u_{n}\right) g\left(u_{n}-u_{m}\right)-F(v) g(u-\delta \varepsilon) d u_{n}}{u_{n}-u_{m}-\delta \varepsilon}+ \\
& +F\left(u_{m}+\delta \varepsilon\right) g(\delta \varepsilon) \ln \left|\frac{w-\delta \varepsilon}{w+\delta \varepsilon}\right|,
\end{aligned}
$$

where $\mathrm{F}$ represents the function $\mathrm{C}$ or $\mathrm{D}$.

We use a schematic form for the phonon spectrum[9] satisfying $\rho\left(-w_{q}\right)=\rho\left(w_{q}\right)$ with a cutoff frequency $w_{c}$ :

$$
g\left(w_{q}\right)=\left\{\begin{array}{lc}
2\left(\frac{g_{1}}{w_{c}}\right)^{2} w_{q}+C g_{2}^{2} w_{q} w_{q} & 0<\left|w_{q}\right|<w_{1}, \\
2\left(\frac{g_{1}}{w_{c}}\right)^{2} w_{q}+C g_{2}^{2} \frac{\left(w_{2}-\mid w_{q}\right)^{n} w_{q}}{\left|w_{q}\right|} & w_{1}<\left|w_{q}\right|<w_{2}, \\
2\left(\frac{g_{1}}{w_{c}}\right)^{2} w_{q} & w_{2}<\left|w_{q}\right|<w_{c},
\end{array}\right.
$$

where

$$
\begin{gathered}
n=2 \log \left(w_{1}\right) / \log \left(w_{2}-w_{1}\right) \\
C=\left[\frac{w_{1}^{3}}{3}+\frac{\left(w_{2}-w_{1}\right)^{n+1} w_{q}}{n+1}\right]^{-1} .
\end{gathered}
$$

With this expression for $\mathrm{n}$, the function $g\left(w_{q}\right)$ is continuous at $w_{q}=w_{1}$ and the expression for $\mathrm{C}$ assures that

$$
\int_{u-w}^{u+w} \frac{\rho\left(w_{q}\right)}{2 w_{q}} d w_{q}=1
$$

is satisfied separately by the acoustical and the optical contributions. When $g_{2}=0$ the above expressions correspond to a Debye spectrum from $w_{q}=0$ to $w_{q}=w_{c}$; $g_{2} \neq 0$ adds an optical contribution from $w_{q}=0$ to $w_{q}=w_{2}$ peaked at $w_{q}=w_{1}$.

\section{Results and Discussions}

We have taken here $E_{A}^{0}=0, E_{E}^{0}=2 t_{0}, E_{A}^{1}=$ $E_{10}+t_{0}-t_{1}$ and $E_{E}^{1}=E_{10}+t_{0}+t_{1}$ (see Fig. 1), with the following values of the parameters $E_{10}=10 \mathrm{meV}$, $2 t_{0}=35 \mu \mathrm{eV}, 2 t_{1}=-1 \mathrm{meV}, w_{1}=5 \mathrm{meV}, w_{2}=8 \mathrm{meV}$ and $w_{c}=30 \mathrm{meV}$, and four combinations of the remaining parameters: (a) $g_{1}=1.5 \mathrm{meV}$ and $g_{2}=1.5 \mathrm{meV}$, (b) $g_{1}=2.0 \mathrm{meV}$ and $g_{2}=1.5 \mathrm{meV}$, (c) $g_{1}=2.0 \mathrm{meV}$ and $g_{2}=2.0 \mathrm{meV}$, (d) $g_{1}=2.0 \mathrm{meV}$ and $g_{2}=2.5 \mathrm{meV}$. These values of $g_{1}$ and $g_{2}$ are significantly greater than those selected in the reference[9], but are those for which the vertex part begins to show appreciate effects.
To simulate a non-zero instrumental resolution we have added $1.5 \mu \mathrm{eV}$ to the imaginary part of the self-energies. The computation were performed with a FORTRAN77 program with a typical runtime of 1 hour in a DEC 2000 Alpha-XP workstation. Fig. 4 shows the spectral density for different temperatures corresponding to the case (c) with the vertex part already included. The line intensity has been calculated taking $Z=Z_{r} Z_{v}$, an approximation which does not affect the lineshift or the linewidth behavior.

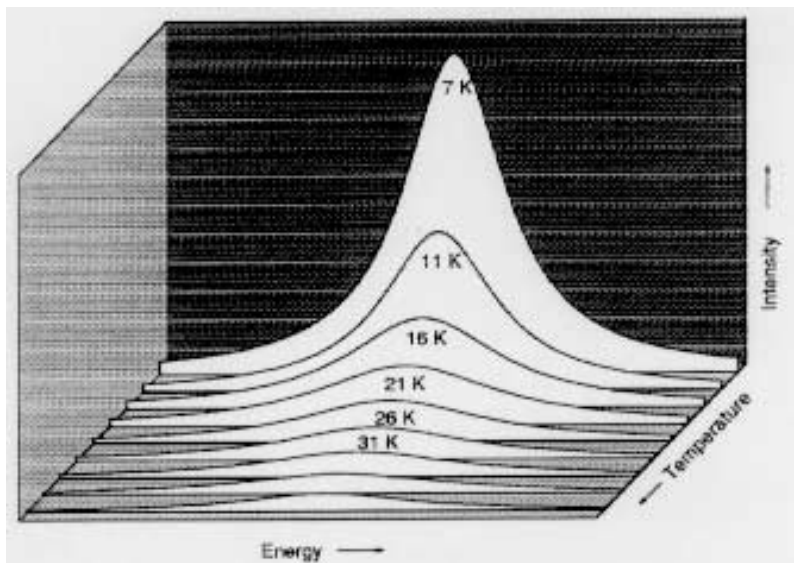

Figure 4. Spectral lines for different temperatures(35K, 40K and $45 \mathrm{~K}$ for the three lowest lines).

We see the tendency of the absorption line to broaden (and ultimately it will merge with the elastic and the emission lines) as the temperature increases. Fig. 5 shows the typical form of the real and of the imaginary parts of the functions $C_{A}^{1}(u)$ and $D_{A}^{1}(u)$. Each of these functions reveals two intervals where their values are significantly different from zero, the two intervals corresponding to quite different energy scales. At the same time, the height of the maximum of each 
function in the two intervals keeps an inverse relation with the corresponding energy scale, a fact not reflected in the figure for convenience. Of course, only after an exhaustive search it was possible to uncover the full behavior of these functions.

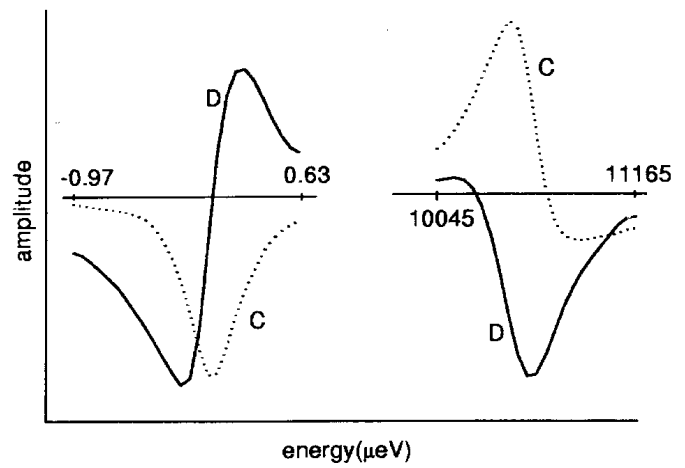

Figure 5. Typical aspect of the functions $C_{A}^{1}(\varepsilon)$ and $D_{A}^{1}(\varepsilon)$ as discussed in the text.

Fig. 6 to Fig. 9 contain the main results of our calculation, corresponding to the cases (a), (b), (c), and (d) cited above. They show the graphs of the logarithm of the lineshift $\Delta$ (dashed lines) and of the linewidth $\Gamma$ (solid lines) as a function of $1 / T$. The effect of the vertex part correction is also shown, and is represented in each figure by the piece of solid line that seems to extend the straight line character of the lineshift behavior. To construct these graphs we have used the following procedure: for each temperature we fitted the points along the curve $\rho(w)$ near half height at each side of the maximum and the points near the maximum to a polynomial function. For each section of the curve we worked with 50 out of a total of 600 points used to generate $\rho(w)$, producing fittings with standard deviation always less than 1.5\%. By other hand, trying to fit all the 600 points to a single Lorentzian curve, we always found standard deviation between $12 \%$ and $16 \%$, this because the actual form of $\rho(w)$ is of a distorted Lorentzian.

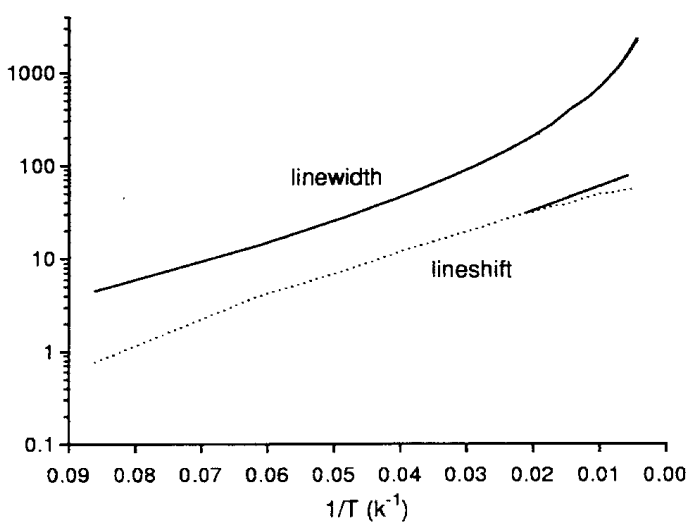

Figure 6. Plot corresponding to the case (a) (see text).

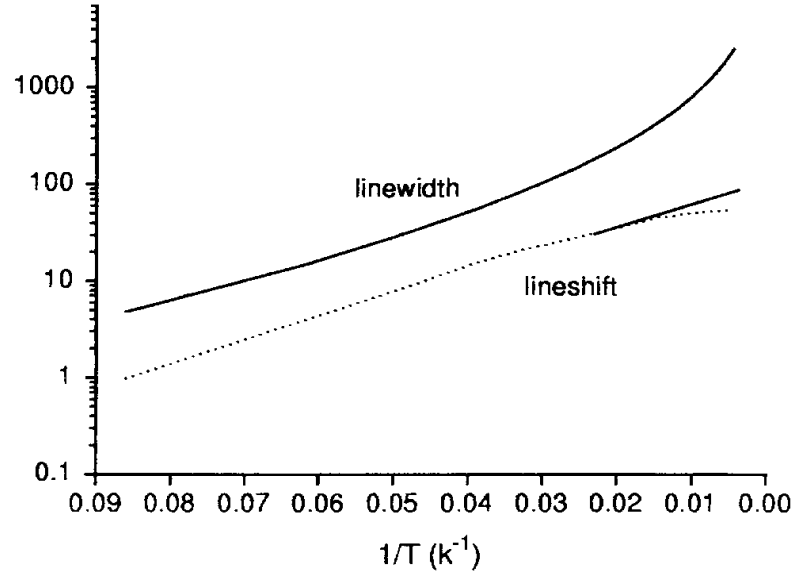

Figure 7. Plot corresponding to the case (b) (see text).

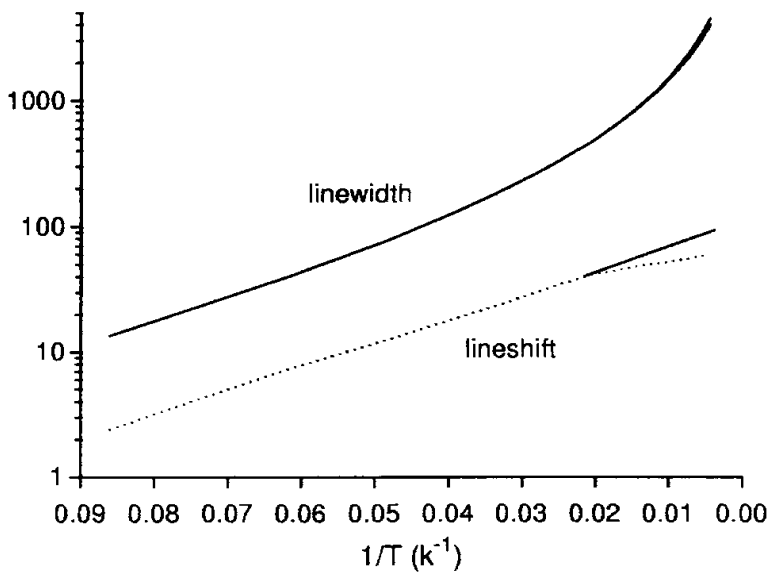

Figure 8. Plot corresponding to the case (c) (see text).

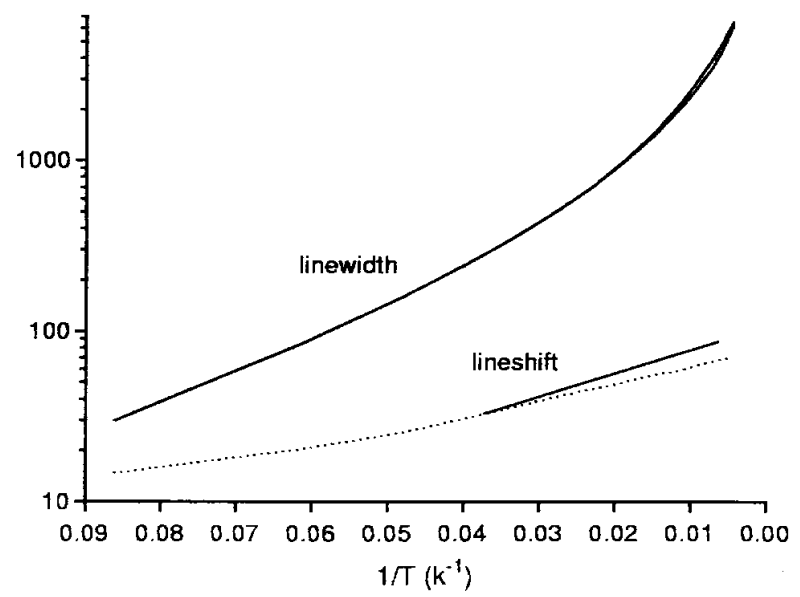

Figure 9. Plot corresponding to the case (d) (see text).

From Fig. 6 to Fig. 7 we increase the weight of the acoustical phonons, and from Fig. 8 to Fig. 9 we increase the weight of the optical phonons. Clearly, the vertex part changes very little the behavior of the linewidth, at least in the range of temperatures considered here. This behavior is of a straight line in the $\log \Gamma-1 / T$ plot at low temperatures. If this behavior 
is present also at higher temperatures, as found in the reference [10], we see here that the corresponding slopes are much larger than those for low temperatures. In the case of the lineshift, the vertex part correction begins to be effective only at temperatures beyond which probably the contribution of higher librational levels than those corresponding to $\mathrm{m}=0$ and $\mathrm{m}=1$ considered here becomes important. The correction increases with increasing weight of the optical phonons, and it partially compensates the tendency of the lineshift to deviate from a straight line in the $\log \Gamma-1 / T$ plot at higher temperatures. Interesting in the plots of the lineshift is the systematic occurrence of two straight lines of different slopes at different temperature intervals. As we checked also for other values of the parameters, the straight line at lower temperatures has a smaller slope than that at higher temperatures when the weight of the acoustical phonons is greater than that of the optical phonons, and conversely when the weight of the optical phonons is greater than that of the acoustical phonons. Such change of slope in the lineshift behavior is much smaller and occurs at lower temperatures than the equivalent one that, according the results in the reference[10], is possible to take place in the linewidth behavior.

Our results are qualitatively similar to those obtained in previous works $[9,11]$. Taking into account the differences in the phonon spectrum, they also reveal a temperature dependence of the INS spectral lines that is rather insensitive to the details of the phonon spectrum. This is particularly true in our results for the lineshift behavior, and in remarkable agreement with the more recent experimental observation[2]. Since we have not worked with explicit expressions for $\Gamma$ nor $\Delta$, it is difficult to access the physical reason for some of the results in the present calculation and in particular to discuss the influence of the phonon spectrum. Anyway, we think that any further advance in this problem will only happen on quantitative aspects and only with the use of more realistic phonon spectra. As a final remark, one sees that the vertex part enters as weight factors to the curves $Y_{A}^{0}\left(\varepsilon_{E}^{0}-w\right)$ and $Y_{E}^{0}\left(\varepsilon_{A}^{0}+w\right)$ in the expression of $\rho(w)$. These factors represent corrections to the values $U_{A}^{0}\left(\varepsilon_{E}^{0}-w\right)=1$ and $U_{E}^{0}\left(\varepsilon_{A}^{0}+w\right)=1$, and the fact that they do not contribute significantly to the linewidth behavior means that this contribution is proportional to $w$ with nearly the same proportionality constant for both curves. In fact, we succeeded to simulate ours graphs with a weighting of $[1+\alpha(T) w]$, with $\alpha(T)$ as an adjustable parameter that increases with temperature. This suggests that the first order term of an expansion for the vertex part would be sufficient.

\section{Acknowledgments}

We are grateful to Dr. Wiebe Geertsma for his comments and suggestions. We are also grateful to CAPES/COPLAG-ES for financial support.

\section{References}

[1] A. Wurger, J. Phys. Cond. Matter 1, 1673 (1989).

[2] A. Buekenhoudt, L. Van Gerven, Phys. Rev. B 46, 5377 (1992).

[3] P. S. Allen, J. Phys. C: Solid St. Phys. 7, L22 (1974).

[4] S. Clough, J. Phys. C: Solid St. Phys. 18, L1 (1985).

[5] J. M. F. Gunn, J. Phys. C: Solid St. Phys. 18, L357 (1985).

[6] S. Clough and J.R. Hill, J. Phys. C: Solid St. Phys. 9, L645 (1976).

[7] A. Huller, Z. Phys. B - Cond. Matter 36, 215 (1980).

[8] A. C. Hewson, J. Phys. C: Solid St. Phys. 15, 3841 (1982).

[9] A. C. Hewson, J. Phys. C: Solid St. Phys. 15, 3855 (1982).

[10] M. W. G. Whittall, G. A. Gehring, J. Phys. C: Solid St. Phys. 20, 1619 (1986).

[11] A Wurger, Z. Phys. B - Cond. Matter 76, 65 (1989).

[12] H. Keiter, G.. Morandi, Phys. Rep. 109, 227 (1984).

[13] J. Colmenero, R. Mukhopadhyay, A. Alegría, B. Frick, Phys. Rev. Lett. 80, 2350 (1998).

[14] J. Peternelj, T. Kranjc, M.M. Pintar, Phys. Rev B 54, 955 (1996).

[15] G. Voll, Physica B 202, 239 (1994).

[16] Changho Choi and Mik M. Pintar, Phys. Rev. Lett. 76, 527 (1996).

[17] C.H. Choi and M.M. Pintar, J. Chem. Phys. 109, 5542 (1998). 